Power Systems and Complexes

Энергетические системы и комплексы

DOI: $10.17516 / 1999-494 X-0359$

УДК 621.316.016.25

\title{
Statistical Multimode Accounting in the Problem of Optimal Reactive Load Compensation when Constructing Smart Grids
}

\author{
Alexey A. Gerasimenko and Roman V. Belyaevsky*b \\ ${ }^{a}$ Siberian Federal University \\ Krasnoyarsk, Russian Federation \\ ${ }^{b}$ T.F. Gorbachev Kuzbass State Technical University \\ Kemerovo, Russian Federation
}

Received 14.09.2021, received in revised form 20.10.2021, accepted 21.11.2021

\begin{abstract}
The article deals with the development prospects for smart grids. An approach to optimizing the modes of electric power systems in terms of reactive power is presented in detail. The solution of this problem considers the multimodality, determination of the integral characteristics of the modes, etc. Analytical modeling of load changes by means of the method of factor analysis allows to reduce the amount of information drastically without a significant loss of the accuracy of the obtained solutions. For this purpose, the actually correlated electrical loads of various nodes of electric power systems are represented in the form of a linear combination of independent random variables, namely, generalized load graphs. It is shown that the inclusion of multimode by orthogonal graphs results in a significant simplification of the solution of multimode problems. The choice of the dependent and independent variables composition when solving the optimization problem with the power consumption modes has a fundamental effect both on the modeling of constraints, the formation of the reduced gradient and the main calculation expressions, and on the speed of the optimization search as a whole.
\end{abstract}

Keywords: digital energy, smart grid, reactive power, power losses, statistical multimode accounting, generalized load graph, nodal voltage equation.

Citation: Gerasimenko, A.A., Belyaevsky, R. V. Statistical multimode accounting in the problem of optimal reactive load compensation when constructing smart grids, J. Sib. Fed. Univ. Eng. \& Technol., 2021, 14(8), 886-893. DOI: 10.17516/1999494X-0359

(C) Siberian Federal University. All rights reserved

This work is licensed under a Creative Commons Attribution-Non Commercial 4.0 International License (CC BY-NC 4.0).

* Corresponding author E-mail address: brv.egpp@kuzstu.ru 


\title{
Статистический учет многорежимности
}

\section{в задаче оптимальной компенсации реактивных нагрузок при построении интеллектуальных \\ электроэнергетических систем}

\author{
А. А. Герасименко ${ }^{\mathbf{a}}$, Р. В. Беляевский ${ }^{0}$ \\ ${ }^{a}$ Сибирский федеральный университет \\ Российская Федераиия, Красноярск \\ ${ }^{6}$ Кузбасский государственный технический университет \\ имени Т. Ф. Горбачева \\ Российская Федерация, Кемерово
}

\begin{abstract}
Аннотация. В статье рассмотрены перспективы развития интеллектуальных электроэнергетических систем. Подробно представлен подход к оптимизации режимов электроэнергетических систем по реактивной мощности. Решение данной задачи требует учета многорежимности, определения интегральных характеристик режимов и др. Аналитическое моделирование изменения нагрузок методом факторного анализа позволяет резко снизить объем информации без существенной потери точности получаемых решений. Для этого реально коррелированные электрические нагрузки различных узлов электроэнергетических систем представляются в виде линейной комбинации независимых случайных величин, а именно обобщенных графиков нагрузок. Показано, что учет многорежимности ортогональными графиками приводит к значительному упрощению решения многорежимных задач. Выбор состава зависимых и независимых переменных при решении задачи оптимизации с учетом режимов электропотребления оказывает основополагающее влияние как на моделирование ограничений, формирование приведенного градиента и основных расчетных выражений, так и на скорость оптимизационного поиска в целом.
\end{abstract}

Ключевые слова: цифровая энергетика, интеллектуальная энергосистема, реактивная мощность, потери электроэнергии, статистический учет многорежимности, обобщенный график нагрузки, уравнение узловых напряжений.

Цитирование: Герасименко, А.А. Статистический учет многорежимности в задаче оптимальной компенсации реактивных нагрузок при построении интеллектуальных электроэнергетических систем / А. А. Герасименко, Р. В. Беляевский // Журн. Сиб. федер. ун-та. Техника и технологии, 2021, 14(8). С. 886-893. DOI: 10.17516/1999-494X-0359

\section{Введение}

В настоящее время в Российской Федерации активно реализуется политика перехода к цифровой экономике. В 2019 г. Правительством Российской Федерации была разработана национальная программа «Цифровая экономика Российской Федерации», утвержденная протоколом заседания президиума Совета при Президенте Российской Федерации по стратегическому развитию и национальным проектам от 04.03.2019 № 7, началось формирование отраслевых программ цифрового перехода, в том числе в сфере энергетики. При этом цифровую энергетику следует рассматривать как неотъемлемую составляющую цифровой экономики, особым предметом которой является экономическая деятельность, коммерческие транзакции и профессиональные взаимодействия, построенные на принципах использования информационнотелекоммуникационных технологий.

$$
-887-
$$


Так, в [1] сделаны выводы, что существующий технологический уклад в электроэнергетике достиг предела своей эффективности и в перспективе пяти лет в ряде сфер, где потребители предъявляют более высокие требования к надежности, качеству, доступности, экологичности электроснабжения, будет иметь меньшую конкурентоспособность по сравнению с решениями цифровой энергетики. При этом характерным признаком цифровой энергетики является ее киберфизический характер [2]. Он становится возможен, когда так называемые интеллектуальные системы начинают формировать и использовать цифровые модели физического мира. Именно это обеспечивает самостоятельность принятия ими решений в режиме, близком к реальному времени. Для этого они должны руководствоваться не жесткими стандартами, а заданными алгоритмами, целевыми функциями и цифровыми моделями реальных электроэнергетических систем.

\section{Методы исследования}

В этих условиях оптимизация режимов электроэнергетических систем (ЭЭС) по реактивной мощности позволяет значительно повысить эффективность их функционирования. Решение названной задачи в общей проблеме оптимального функционирования интеллектуальных ЭЭС требует учета многообразия их режимов (многорежимности), определения интегральных характеристик режимов, таких как потери электрической энергии, диапазоны изменения режимных параметров и др. Непосредственный переход к многорежимным моделям резко увеличивает размерность и трудоемкость решения оптимизационной задачи.

В [3, 4] реализован стохастический подход учета многорежимности, вызванный изменением электрических нагрузок, для целей анализа и оптимизации режимов ЭЭС по реактивной мощности, позволяющий учесть стохастическую природу значительной части информации о нагрузках. Аналитическое моделирование изменения нагрузок методом факторного (компонентного) анализа дает возможность резко снизить объем информации без существенной потери точности получаемых решений. На основе полученной статистической факторной модели нагрузок реально коррелированные электрические нагрузки различных узлов ЭЭС представляются в виде линейной комбинации небольшого количества М (достаточно принять равным трем-четырем) независимых случайных величин (коэффициентов) - главных факторов, именуемых обобщенными (ортогональными) графиками нагрузок (ОГН) $[5,6]$.

ОГН представляют собой совокупность статистически независимых базисных векторов, ориентированных так, что каждый из них отражает большую часть связи исходной совокупности графиков нагрузок, вносит наибольший вклад в дисперсию исходных переменных. Выделенные факторные модели отражают общие закономерности, основные свойства изменения конфигурации электрических нагрузок, обладают свойствами универсальности, придают статистическому методу и в целом процессу моделирования многорежимности свойства линейности и аддитивности и позволяют с достаточной точностью восстановить исходные параметры изменения нагрузок на рассматриваемом временном интервале [7].

\section{Результаты и их обсуждение}

В случае моделирования графиков нагрузки методом главных компонент на основе представительной выборки $N$ исходных графиков нагрузок определяется матрица корреляционных 
моментов (МКМ) и выделяются М максимальных собственных чисел $\lambda_{i}$ и соответствующие им собственные векторы $v_{i}$ со следующим построением ОГН:

$$
\mathrm{G}_{k j}=\sum_{i=1}^{N} v_{k i}^{\prime} \Delta P_{i j}+\sum_{i=1}^{N} v_{k i}^{\prime \prime} \Delta Q_{i j} \quad j=\overline{1, d}, k=\overline{1, M}
$$

где $\mathrm{v}_{k i}^{\prime}, \mathrm{v}_{k i}^{\prime \prime}$ - компоненты собственного вектора $\overline{\mathrm{v}}_{k} \mathrm{MKM;} \Delta P_{i j}, \Delta Q_{i j}$ - компоненты $j$ центрированных графиков активной $P_{i}$ и реактивной $Q_{i}$ нагрузок узла $i$ с $d$ интервалами постоянства.

Данное статистическое преобразование МКМ позволяет достаточно точно моделировать исходные графики электрических нагрузок $P_{i j}, Q_{i j}$ с помощью известных математических ожиданий $M P_{i}, M Q_{i}$ и моделируемых отклонений нагрузок от математических ожиданий в виде М линейных комбинации статистически устойчивых ОГН:

$$
P_{i j}=M P_{i}+\sum_{k=1}^{M} v_{k i}^{\prime} \mathrm{G}_{k j} ; Q_{i j}=M Q_{i}+\sum_{k=1}^{M} v_{k i}^{\prime \prime} \mathrm{G}_{k j}, i=\overline{1, N}, j=\overline{1, d}
$$

Нагрузочные потери электроэнергии получены в виде суммы основной составляющей $M \Delta E$, определяемой для режима средних нагрузок, и дисперсионной составляющей $\sigma \Delta E$, учитывающей отклонение нагрузок от средних значений:

$$
\begin{aligned}
& \Delta E=M \Delta E+\sigma \Delta E=\Delta P(M \bar{V}, M \bar{\delta}) T+ \\
& +\left[\frac{1}{2} \sum_{i=1}^{N} \sum_{j=1}^{N} k\left(V_{i} V_{j}\right) \frac{\partial^{2} \Delta P}{\partial V_{i} \partial V_{j}}+\sum_{i=1}^{N} \sum_{j=1}^{N} k\left(V_{i j} \delta_{j}\right) \frac{\partial^{2} \Delta P}{\partial V_{i} \partial \delta_{j}}+\right. \\
& \left.+\frac{1}{2} \sum_{i=1}^{N} \sum_{j=1}^{N} k\left(\delta_{i} \delta_{j}\right) \frac{\partial^{2} \Delta P}{\partial \delta_{i} \partial \delta_{j}}\right] T,
\end{aligned}
$$

где $\Delta P(M \bar{V}, M \bar{\delta}) k\left(V_{i} V_{j}\right) k\left(V_{i} \delta_{j}\right) k\left(\delta_{i} \delta_{j}\right)$ - потери мощности, корреляционные моменты, вычисленные для модулей $M V M \delta$ напряжений в точке, соответствующей математическим ожиданиям нагрузок; $\frac{\partial^{2} \Delta P}{\partial V_{i} \partial V_{j}} \frac{\partial^{2} \Delta P}{\partial V_{i} \partial \delta_{j}} \frac{\partial^{2} \Delta P}{\partial \delta_{i} \partial \delta_{j}}-$ вторые производные выражения потерь мощности по соответствующим переменным, вычисленные относительно той же точки; $N$ - количество узлов в схеме без балансирующего.

Статистическая модель электрических нагрузок позволяет перейти от громоздких задач определения интегральных характеристик и оптимизации, переменными которой являются часовые (поинтервальные) нагрузки, к задаче, параметрами которой выступают математические ожидания нагрузок и небольшое М число ОГН, моделирующих отклонения нагрузки от математического ожидания. Показано, что учет многорежимности ортогональными графиками приводит к значительному упрощению решения многорежимных задач. Размерность названных задач, объем вычислений резко уменьшаются. Для определения интегральных характеристик необходимо выполнить одно решение нелинейной системы уравнений узловых напряжений для режима математических ожиданий нагрузок и М решений систем алгебраических уравнений с постоянной матрицей Якоби. Решение данной оптимизационной задачи сводится к ее стандартной форме - задаче нелинейного математического программирования, для решения которой применен наиболее эффективный и широко апробированный метод приведенного

$$
-889-
$$


градиента (МПГ), общая структура которого описана в известных работах Л. А. Крумма, например [8] и др.

МКМ напряжений получают на основе системы уравнений, представляемой аналогично линеаризованным уравнениям узловых напряжений (УУН):

$$
\left[\begin{array}{c:c}
\partial P_{i} / \partial \delta_{j} & \partial P_{i} / \partial V_{j} \\
\hdashline \partial Q_{i} / \partial \delta_{j} & \partial Q_{i} / \partial V_{j}
\end{array}\right]\left[\begin{array}{l}
\Delta \overline{\delta_{i}} \\
\Delta \overline{V_{i}}
\end{array}\right]=\left[\begin{array}{l}
\Delta \overline{P_{i}} \\
\Delta \overline{Q_{i}}
\end{array}\right], i, j=\overline{1, N},
$$

где $\Delta \mathrm{P}_{i}, \Delta Q_{i}, \Delta V_{i}, \Delta \delta_{i}-$ отклонения активных, реактивных мощностей, модулей, фаз напряжений узлов от своих математических ожиданий.

Поскольку отклонения напряжений и мощностей от своих математических ожиданий приближенно связаны системой УУН (4), центрированные случайные параметры (изменения фаз и модулей напряжений) $\Delta \delta_{i}, \Delta V_{i}$, так же как и величины $\Delta P_{i}, \Delta Q_{i}$, аналогично (2) представляются линейными комбинациями ОГН:

$$
V_{i j}=M V_{i}+\sum_{k=1}^{M} \gamma_{k i}^{\prime \prime} \mathrm{G}_{k j} \quad \delta_{i j}=M \delta_{i}+\sum_{k=1}^{M} \gamma_{k i}^{\prime} \mathrm{G}_{k j} i=\overline{1, N}, . j=\overline{1, d}
$$

После подстановки отклонений параметров режима из выражений (2) и (5) в систему (4) коэффициенты $\gamma_{k i}^{\prime}, \gamma_{k i}^{\prime \prime}$, моделирующие отклонения фаз и модулей напряжений от средних значений, вычисляются из решения уравнений, эквивалентных системе линеаризованных УУН

$$
[J] \times\left[\begin{array}{l}
{\overline{\gamma^{\prime}}}_{k i} \\
{\overline{\gamma^{\prime \prime}}}_{k i}
\end{array}\right]=\left[\begin{array}{l}
{\overline{v^{\prime}}}_{k i} \\
{\overline{v^{\prime \prime}}}_{k i}
\end{array}\right], k=\overline{1, M} i=\overline{1, N},
$$

где коэффициенты $v_{k i}^{\prime} \gamma_{k i}^{\prime} v_{k i}^{\prime}, v_{k i}^{\prime \prime}$ определяются с помощью исходных (восстановленных) графиков нагрузок и ОГН согласно (2) формулами вида

$$
v_{k i}^{\prime}=\frac{1}{d} \sum_{j=1}^{d} \mathrm{G}_{k j} \Delta P_{i j} ; v_{k i}^{\prime \prime}=\frac{1}{d} \sum_{j=1}^{d} \mathrm{G}_{k j} \Delta Q_{i j} k=\overline{1, M} i=\overline{1, N} .
$$

Стохастическая модель графиков нагрузок позволяет выразить элементы МКМ напряжений и мощностей с помощью моделирующих коэффициентов

$$
\begin{aligned}
& k\left(\delta_{i} \delta_{j}\right)=\sum_{k=1}^{M} \gamma_{k i}^{\prime} \gamma_{k j}^{\prime} ; k\left(V_{i} \delta_{j}\right)=\sum_{k=1}^{M} \gamma_{k i}^{\prime \prime} \gamma_{k j}^{\prime} k\left(V_{i} V_{j}\right)= \\
& =\sum_{k=1}^{M} \gamma_{k i}^{\prime \prime} \gamma_{k j}^{\prime \prime} \sigma^{2} \delta_{i}=\sum_{k=1}^{M} \gamma_{k i}^{\prime 2} \sigma^{2} V_{i}=\sum_{k=1}^{M} \gamma_{k i}^{\prime \prime 2}
\end{aligned}
$$

Аналогичные выражениям (8) для элементов МКМ мощностей определяются в виде

$$
\begin{aligned}
& k\left(P_{i} P_{j}\right)=\sum_{k=1}^{M} v_{k i}^{\prime} v_{k j}^{\prime}, k\left(P_{i} Q_{j}\right)=\sum_{k=1}^{M} v_{k i}^{\prime} v_{k j}^{\prime \prime}, k\left(Q_{i} Q_{j}\right)= \\
& =\sum_{k=1}^{M} v_{k i}^{\prime \prime} v_{k j}^{\prime \prime}, \sigma^{2}\left(Q_{i}\right)=\sum_{k=1}^{M}\left(v_{k i}^{\prime \prime}\right)^{2}
\end{aligned}
$$

С учетом корреляционных моментов модулей и фаз напряжений (8) традиционное выражение нагрузочных потерь электроэнергии (3) представляется в виде

$$
-890-
$$




$$
\begin{aligned}
& \Delta E=[\Delta P(M \bar{V}, M \bar{\delta})+\sigma \Delta P] T= \\
& =\left[\Delta P(M \bar{V}, M \bar{\delta})+\frac{1}{2} \sum_{k=1}^{M} \sum_{i=1}^{N} \sum_{j=1}^{N} \gamma_{k i}^{\prime \prime} \gamma_{k j}^{\prime \prime} \frac{\partial^{2} \Delta P}{\partial V_{i} \partial V_{j}}+\right. \\
& \left.+\sum_{k=1}^{M} \sum_{i=1}^{N} \sum_{j=1}^{N} \gamma_{k i}^{\prime \prime} \gamma_{k j}^{\prime} \frac{\partial^{2} \Delta P}{\partial V_{i} \partial \delta_{j}}+\frac{1}{2} \sum_{k=1}^{M} \sum_{i=1}^{N} \sum_{j=1}^{N} \gamma_{k i}^{\prime} \gamma_{k j}^{\prime} \frac{\partial^{2} \Delta P}{\partial \delta_{i} \partial \delta_{j}}\right] T .
\end{aligned}
$$

Потери электроэнергии холостого хода трансформаторов $N_{T}$ с проводимостями $g_{i}^{T}$ уточняются в исходном и оптимальном режимах в соответствии с полученными графиками напряжений (4):

$$
\Delta E_{\mathrm{X}}=\sum_{i=1}^{N_{T}} g_{i}^{T} \int_{0}^{T} V_{i}^{2}(t) d t \approx \sum_{i=1}^{N_{T}} \sum_{j=1}^{d} g_{i}^{T} V_{i j}^{2} t_{j} \approx T \sum_{i=1}^{N_{T}} g_{i}^{T} M V_{i}^{2} .
$$

В процессе решения эксплуатационной задачи определяется минимум целевой функции суммарных потерь электрической энергии (10), (11) при балансовых нелинейных ограничениях в виде равенств (УУН) для математических ожиданий параметров режима и простых ограничениях типа неравенств

$$
Q_{i}^{\min } \leq Q_{i} \leq Q_{i}^{\max }, i=\overline{1, G} ; V_{i}^{\min } \leq V_{i} \leq V_{i}^{\max }, i=\overline{1, N}
$$

где $G$ - число узлов с источниками реактивной мощности.

Ограничения (12) должны выполняться на всем временном интервале, т. е. для каждого режима. Для этого предложены два способа контроля: 1) моделирование с помощью ОГН графиков реактивной мощности (2) и напряжений (4) на каждом шаге оптимизации и проверка соответствия ограничениям (11);2) вычисление расчетных диапазонов изменения рассматриваемых параметров, которые с учетом дисперсий (8), (9) определяются неравенствами Чебышева.

\section{Заключение}

Таким образом, целевая функция суммарных потерь электроэнергии формируется основной составляющей, вычисляемой для средних нагрузок, и дисперсионной составляющей, выраженной обобщающими характеристиками отклонения параметров режима от средних значений. Выбор состава зависимых и независимых переменных при решении задачи оптимизации с учетом режимов электропотребления оказывает основополагающее влияние как на моделирование ограничений, формирование приведенного градиента и основных расчетных выражений, так и на скорость оптимизационного поиска в целом. В случае, когда в качестве независимых переменных принимаются математические ожидания реактивных мощностей источников и коэффициенты, моделирующие отклонения от их средних значений, вычисление приведенного градиента в основном сводится к $\mathrm{M}+1$ решению систем с постоянной матрицей (при пассивности ограничений - с единичной матрицей), решение которых проще, чем решение системы линеаризованных уравнений узловых напряжений.

Практическая реализация алгоритма оптимизации может быть осуществлена благодаря его малой трудоемкости. Для этого достаточно выполнить один цикл оптимизационных расчетов по математическим ожиданиям нагрузок и решить дополнительно М систем алге- 
браических уравнений с неизменной матрицей коэффициентов. На основе разработанного стохастического алгоритма МПГ, учитывающего множество режимов, была составлена экспериментальная программа, применение которой на ряде схем ЭЭС показало эффективность предлагаемого метода. Следовательно, данный подход может быть использован при разработке архитектуры интеллектуальных ЭЭС $[9,10]$.

\section{Список литературы / References}

[1] Княгинин В. Н., Холкин Д. В. Цифровой переход в электроэнергетике России: экспертноаналитическийдоклад[Электронныйресурс]-Режимдоступа:https://www.csr.ru/uploads/2017/09/ Doklad_energetika-Web.pdf - Заглавие с экрана. [Knyaginin V.N., Kholkin D. V. Digital Transition in the Electric Power Industry of Russia: Expert and Analytical Report [Electronic resourse] - Access: https://www.csr.ru/uploads/2017/09/Doklad_energetika-Web.pdf

[2] Кобец Б. Б., Волкова И. О. Инноваиионное развитие электроэнергетики на базе концепuии Smart Grid, М.: ИАЦ Энергия, 2010. 208 c. [Kobets B. B., Volkova I. O. Innovative development of the electric power industry based on the Smart Grid concept, Moscow: EAC Energy, 2010. 208 p. (in Russian)]

[3] Герасименко А.А., Нешатаев В.Б. Оптимальная компенсация реактивной мощности в системах распределения электрической энергии, Красноярск: СФУ, 2012. 218 с. [Gerasimenko A.A., Neshataev V.B. Optimal Reactive Power Compensation in electrical Power Distribution Systems, Krasnoyarsk: Siberian Federal University, 2012. 218 p. (in Russian)]

[4] Беляевский Р. В. Разработка многоуровневой модели оптимизации размещения компенсирующих устройств в электрических сетях территориальных сетевых организаций. Вестн. Кузбас. гос. техн. ун-та, 2017, 5, 145-149 [Belyaevsky R. V. Development of a Multi-Level Model for Optimizing the Placement of Compensating Devices in the Electrical Networks of Territorial Grid Organizations, Bulletin of the KuzSTU, 2017, 5, 145-149 (in Russian)]

[5] Buran A., Nizkodubov G., Pryahin I. Smart Grid Technologies: Trends and Perspectives, MATEC Web of Conferences, 2016, 91, 01054 (in Russia).

[6] Shulgin I. V., Gerasimenko A. A., Su. Q.Z. Modified Stochastic Estimation of Load Dependent Energy Losses in Electric Distribution Network, International Journal of Electrical Power \& Energy Systems, 2012, 43(1), 325-332 (in Netherlands).

[7] Герасименко А.А., Шульгин И.В. Стохастический метод расчета нагрузочных потерь электроэнергии в распределительных электрических сетях. Электрические станщии, 2013, 4, 44-59 [Gerasimenko A. A., Shulgin I. V. Stochastic method for calculating load losses of electricity in electrical distribution networks, Power stations, 2013, 4, 44-59 (in Russian)]

[8] Крумм Л. А. Методы приведенного градиента при управлении электроэнергетическими системами, Новосибирск.: Наука, 1977. 368 с. [Krumm L.A. Reduced Gradient Methods for Controlling Electric Power Systems, Novosibirsk: Nauka, 1977. 368 p. (in Russian)]

[9] Герасименко А.А., Нешатаев В.Б. Программа оптимизации режимов электроэнергетических систем по реактивной мощности на интервале времени «ORESA». Патент России № 2012610439, 2012 [Gerasimenko A.A., Neshataev V.B. Program for Optimization of Modes of Electric Power Systems in Terms of Reactive Power in the Time Interval «ORESA». Russian Patent No. 2012610439, 2012 (in Russian)] 
[10] Belyaevsky R, Gerasimenko A. Development of Mechanisms for Active-Adaptive Control of Reactive Power Based on Intelligent Electrical Networks, E3S Web of Conferences, 2020, 209, 02004 (in Russia) 